\title{
Strong cosmic censorship for solutions of the Einstein-Maxwell field equations with polarized Gowdy symmetry
}

\author{
Ernesto Nungesser and Alan D Rendall \\ Max-Planck-Institut für Gravitationsphysik, Albert-Einstein-Institut, Am Mühlenberg 1, \\ 14476 Potsdam, Germany
}

Received 29 January 2009, in final form 13 March 2009

Published 29 April 2009

Online at stacks.iop.org/CQG/26/105019

\begin{abstract}
A proof of strong cosmic censorship is presented for a class of solutions of the Einstein-Maxwell equations, those with polarized Gowdy symmetry. A key element of the argument is the observation that by means of a suitable choice of variables the central equations in this problem can be written in a form where they are identical to the central equations for general (i.e. non-polarized) vacuum Gowdy spacetimes. Using this, it is seen that the deep results of Ringström on strong cosmic censorship in the vacuum case have implications for the Einstein-Maxwell case. Working out the geometrical meaning of these analytical results leads to the main conclusion.
\end{abstract}

PACS number: $04.40 . \mathrm{Nr}$

Mathematics Subject Classification: $83 \mathrm{C} 05$, 83C22, 83C75

\section{Introduction}

The Gowdy spacetimes are a class of solutions of the vacuum Einstein equations with two commuting spacelike Killing vectors. Factoring by the symmetry leads to effective equations in one time and one space dimension. To date, the Gowdy spacetimes are one of the best model systems for obtaining a mathematical understanding of the dynamics of inhomogeneous solutions of the Einstein equations. It is known that close analogues of the Gowdy spacetimes exist in the case that the vacuum Einstein equations are replaced by the source-free EinsteinMaxwell equations (see, for instance, [4]). It is thus natural to ask to what extent the known results on the physical properties of Gowdy spacetimes can be generalized to the case with an electromagnetic field. This question is the subject of this paper. When referring to Gowdy spacetimes in the following, the spatial topology is always assumed to be $T^{3}$ (a three-dimensional torus) except where the contrary is stated explicitly.

A variety of deep mathematical results have been obtained concerning Gowdy spacetimes, culminating in the work of Ringström [16, 17, 19]. In particular, it was shown in [19] that strong cosmic censorship holds in the class of Gowdy spacetimes. This means that for generic 
initial data within this class, the corresponding maximal globally hyperbolic development is inextendible. (Precise definitions of these terms are given later in this paper.) A relatively simple special case of the Gowdy spacetimes are those which are polarized. In this case, the main field equation becomes linear and this simplifies many things. In the case of the EinsteinMaxwell equations, it is also possible to make a definition of polarized Gowdy symmetry but in this case the main field equations are no longer linear. In fact, as shown explicitly below, when expressed in suitable variables they are identical to the full (non-polarized) Gowdy equations of the vacuum case. Hence the results of Ringström concerning the solutions of the partial differential equations can be taken over directly. On the other hand, the geometrical and physical meaning of the variables is different in the two cases so that the interpretation of the results is different. This will be worked out in detail in the following. It leads in particular to a result on strong cosmic censorship for solutions of the Einstein-Maxwell equations with polarized Gowdy symmetry (theorem 2).

To put this result into context, it is worth recalling for which matter models strong cosmic censorship has been proved for any inhomogeneous solutions of the Einstein-matter equations. In [7], strong cosmic censorship was proved for solutions of the Einstein-Vlasov system with $T^{2}$ symmetry. The genericity assumption made there excludes the vacuum case. The matter content is used in an essential way. Although it is plausible that the methods of [7] could be extended to many other matter models, they do not suffice to treat the case of a Maxwell field alone. Similar results have been proved for solutions of the Einstein-Vlasov system with spherical symmetry and hyperbolic symmetry in [8]. The results of [7] were extended to the case with a positive cosmological constant in [20]. Strong cosmic censorship has been proved for the plane symmetric Einstein-scalar field system in [21]. For those Gowdy spacetimes (not considered further in the following) with topologies other than $T^{3}$ cosmic censorship has been studied in the polarized case in [5]. The results which have been listed are those existing positive results known to the authors on strong cosmic censorship of this level of generality. They are valid for inhomogeneous solutions of the field equations, with or without matter. They concern solutions which belong to some symmetry class but do not require smallness assumptions on the initial data.

Apart from cosmic censorship, more detailed information is obtained about the qualitative properties of the solutions of the Einstein-Maxwell equations near the initial singularity and at late times. For generic solutions, it is shown that the Kretschmann scalar $R_{\alpha \beta \gamma \delta} R^{\alpha \beta \gamma \delta}$ blows up uniformly on certain regions as the singularity is approached. It is also shown that the spacetimes are geodesically complete towards the future and that they exhibit oscillatory behaviour at late times analogous to that found in [16] for Gowdy spacetimes.

In section 2, definitions of Gowdy and polarized Gowdy symmetry are introduced. These definitions, which make use of discrete symmetries, have not previously been worked out in the literature and so are treated in detail here. In section 3, a number of equations are displayed. They are the basic equations of the class of spacetimes which are of interest in the following. In sections 4 and 5, the main results on the asymptotic behaviour of solutions in the past and future directions respectively are proved. These results are combined in section 6 to give the main result (theorem 2) and some thoughts are presented on how the results of this paper might be generalized in future.

This paper is based in part on the diploma thesis of the first author [12].

\section{Gowdy spacetimes and discrete symmetries}

Let $g_{\alpha \beta}$ be a Lorentzian metric on a four-dimensional manifold $M$ and suppose that $g_{\alpha \beta}$ is a solution of the Einstein equations coupled to some matter model. A symmetry of this solution 
is an isometry of the metric which also preserves the matter fields. The solution is said to have $T^{2}$ (or $U(1) \times U(1)$ ) symmetry if this group acts on $M$ by symmetries of the solution. Think of a solution as being defined by Cauchy data. If the Cauchy data are invariant under a symmetry group and the Einstein equations coupled to the given matter model have a well-posed initial value problem, then there is an associated action of the group on the solution (cf the discussion in section 9.2 of [15]).

Now suppose a spacetime is given where the metric takes the form

$\mathrm{e}^{2(\eta-U)}\left(-\mathrm{d} t^{2}+\mathrm{d} \theta^{2}\right)+\mathrm{e}^{2 U}[\mathrm{~d} x+A \mathrm{~d} y+(G+A H) \mathrm{d} \theta]^{2}+\mathrm{e}^{-2 U} R^{2}[\mathrm{~d} y+H \mathrm{~d} \theta]^{2}$.

Here $\theta, x$ and $y$ are periodic coordinates on $T^{3}$ and the functions $\eta, U, A, G, H$ and $R$ depend only on $t$ and $\theta$. This metric has $T^{2}$ symmetry with the group action generated by the Killing vectors $\frac{\partial}{\partial x}$ and $\frac{\partial}{\partial y}$. This is a form of the metric used in [2]. In that paper, it was shown that in any globally hyperbolic spacetime with $T^{2}$ symmetry and a Cauchy hypersurface diffeomorphic to $T^{3}$ coordinates of this type can be introduced in a neighbourhood of any Cauchy hypersurface.

A metric of the form (1) will be said to have Gowdy symmetry if the transformation which simultaneously maps $x$ to $-x$ and $y$ to $-y$ is an isometry. It follows that $G$ and $H$ must vanish. Conversely, if $G$ and $H$ vanish, the spacetime has this extra symmetry. The terminology comes from the fact that the solutions of the vacuum Einstein equations with this symmetry are precisely the Gowdy spacetimes. A metric with Gowdy symmetry is said to be polarized if the individual transformations mapping $x$ to $-x$ and $y$ to $-y$ are symmetries. This is equivalent to the vanishing of $A$. Next, a more abstract characterization in terms of discrete symmetries will be derived. Let $G_{1}$ be the group of isometries of $\mathbf{R}^{2}$ generated by translations and reflection in the origin. Let the latter transformation be denoted by $R_{1,2} . G_{1}$ is a semidirect product of $\mathbf{R}^{2}$ and $Z_{2}$. Suppose that in a spacetime with $T^{2}$ symmetry the action of $T^{2}$ extends to an action of $G_{1}$ by isometries. Then the coordinate form of the transformation corresponding to $R_{1,2}$ is given up to an overall translation by the transformation which simultaneously sends $x$ to $-x$ and $y$ to $-y$. This follows from the relation $R_{1,2} T_{a, b}=T_{-a,-b} R_{1,2}$ which holds for any translation $T_{a, b}$. To see this, note first that the isometry corresponding to $R_{1,2}$ cannot be a translation. Hence it has a fixed point. Evaluating the relation at that fixed point gives the desired conclusion for the restriction on one orbit. Consider the geodesic in $S$ through that point orthogonal to the orbit. Its intersection with any other orbit must also be a fixed point of $R_{1,2}$. Thus, the necessary translation is the same for all orbits on the initial hypersurface. The same conclusion can then be obtained for any orbit in the spacetime by a similar argument using a geodesic of the spacetime through the fixed point which is orthogonal to the initial hypersurface. In this way, an abstract characterization of Gowdy symmetry in terms of an action of the group $G_{1}$ is obtained. Similarly, polarized Gowdy symmetry can be characterized by the fact that the $T^{2}$ action extends to an action by isometries of the group $G_{2}$ of isometries of $\mathbf{R}^{2}$ generated by translations and the reflections $R_{1}$ and $R_{2}$ in the two Cartesian coordinates. The group $G_{2}$ is a semidirect product of $\mathbf{R}^{2}$ with $Z_{2} \oplus Z_{2}$. The elements $R_{1}$ and $R_{2}$ satisfy the relations $R_{1} T_{a, b}=T_{-a, b} R_{1}$ and $R_{2} T_{a, b}=T_{a,-b} R_{2}$. It follows by the same type of argument as in the previous case that $R_{1}$ and $R_{2}$ can be identified with reflections in $x$ and $y$.

Let us suppose for simplicity that the torus has unit coordinate volume with respect to the coordinates $(x, y)$. Then the area of an orbit of the symmetry group contained in the domain of the above coordinate system is given by $R$. This can be used to extend the definition of $R$ to the whole spacetime by requiring its value at a given point to be equal to the area of the orbit on which that point lies. The function $R$ is called the area radius. It will be assumed in the following that the Maxwell field does not vanish identically so that the spacetime is not 
flat. Recall that the Maxwell field satisfies the dominant energy condition. A proof of this is given in the appendix. It follows from proposition 3.1 of [14] that the gradient of $R$ is always timelike. As a consequence the function $R$ can be used as a time coordinate. This is referred to as an areal time coordinate. It is not a priori clear whether in a given solution there is any level surface of $R$ which is a Cauchy surface. An argument which shows that there is a level surface of this kind is presented in the following section.

Having defined Gowdy and polarized Gowdy symmetry for metrics, the next step is to do the same for solutions of the Einstein-Maxwell equations. To do this, the action of the groups $G_{1}$ and $G_{2}$ must be extended to the Maxwell field. Any diffeomorphism transports any tensor field in a natural way. If the diffeomorphism is an isometry consistency with the Einstein equations requires that the energy-momentum tensor must be transported in this way. It is, however, possible to transport matter fields in a different way and this will be done here. Consider the case that the electromagnetic field is defined by a four-potential $A_{\alpha}$. If $\phi$ is a diffeomorphism, consider the transformation $(g, A) \mapsto\left(\phi_{*} g,-\phi_{*} A\right)$. This leads to the transformation $F \mapsto-\phi_{*} F$ for the electromagnetic field. This ensures that the energymomentum tensor is left invariant. If the action of the group $G_{1}$ is extended so as to transform the electromagnetic field in this way then $A_{0}$ and $A_{1}$ vanish. Denote the remaining components of the potential by $\omega=A_{2}$ and $\chi=A_{3}$. This provides the definition of Gowdy symmetry for the Einstein-Maxwell system. The only non-trivial components of the electromagnetic field are $F_{02}, F_{03}, F_{12}$ and $F_{13}$. Taking instead $G_{2}$ provides the definition of polarized Gowdy symmetry. This does not lead to a direct further restriction on the potential but does further restrict the metric. It will be shown in the following section that the Einstein-Maxwell equations lead to a consistency condition which apparently forces one of the components of the potential to be set to zero in the polarized case.

It would have been possible to use the more straightforward transformation $(g, A) \mapsto$ $\left(\phi_{*} g, \phi_{*} A\right)$ instead of that just introduced. This leads to a different class of solutions of the Einstein-Maxwell equations. In that case, the only non-trivial components of the potential are $A_{0}$ and $A_{1}$. The only component of the electromagnetic field which may be non-vanishing in this case is then $F_{01}$. If fields are allowed which do not arise from a potential then $F_{23}$ may also be non-vanishing. Solutions of the Einstein-Maxwell equations of this type were considered in [22]. It seems that they exhibit oscillatory behaviour near the singularity and are thus more complicated than the solutions considered in the following. This other symmetry class is not considered further in the present work.

\section{Basic equations}

Consider Cauchy data for a solution of the Einstein-Maxwell equations with polarized Gowdy symmetry and suppose that these data have a constant value of $R$. In the solution evolving from these data coordinates exist on a neighbourhood of the initial hypersurface where the metric takes the form (1) with $G=H=A=0$. The function $R$ can be introduced as a new time coordinate, and after that a new spatial coordinate can be introduced so that $g_{01}=0$. Then the transformed metric is of the form (1) with $G=H=A=0$ and $R=t$ except for the fact that $\mathrm{d} t^{2}$ may have been replaced by an expression of the form $\alpha \mathrm{d} t^{2}$ for some function $\alpha(t, \theta)$. Using the fact that for the Maxwell field $T_{0}^{0}=-T_{1}^{1}$ one of the field equations (equation (11.42) on $\mathrm{p} 224$ of [15]) implies that $\alpha_{t}=0$. It is possible, while maintaining the coordinate conditions imposed up to now, to reparametrize the coordinate $\theta$ on the initial hypersurface. Because $\alpha$ does not depend on time, this can be used to impose the condition $\alpha=1$. Introduce new variables as follows: $\lambda=4(\eta-U)+\log t, P=2 U-\log t$. Then the metric takes 
the form

$$
t^{-\frac{1}{2}} \mathrm{e}^{\frac{\lambda}{2}}\left(-\mathrm{d} t^{2}+\mathrm{d} \theta^{2}\right)+t\left(\mathrm{e}^{P} \mathrm{~d} x^{2}+\mathrm{e}^{-P} \mathrm{~d} y^{2}\right) .
$$

In the new coordinates, the original Cauchy hypersurface is not a hypersurface of constant $t$. That there does exist a Cauchy hypersurface of constant $t$ can be shown as follows. The maximal Cauchy development of the initial data corresponds to a certain subset of $(t, \theta)$ space. Suppose that this set has a past boundary point $p$ at which $t$ is strictly positive. By using standard light cone estimates for the Gowdy equations it can be shown that $P, \chi$ and all their derivatives all orders are uniformly bounded on the intersection of the region corresponding to the maximal Cauchy development with a small neighbourhood of $p$. By uniform continuity they extend continuously to $p$. Using arguments as in sections 4 and 5 of [1] or as in [3] it can be concluded that the solution can be extended to a larger region in such a way as to define a globally hyperbolic extension. This contradicts the definition of the maximal Cauchy development. Hence no such boundary point $p$ exists. The solution exists all the way to $t=0$ and hence if $t_{0}$ is any positive number smaller than the minimum of $t$ on the original Cauchy hypersurface then $t=t_{0}$ will be a Cauchy surface of the type whose existence was to be demonstrated.

As explained in the previous section, the only non-vanishing components of the fourpotential defining the Maxwell field are $A_{2}=\omega$ and $A_{3}=\chi$. With these choices the Einstein-Maxwell equations can be computed. The details are given in [12]:

$$
\begin{aligned}
& -P_{t t}-t^{-1} P_{t}+P_{\theta \theta}=-2 t^{-1}\left[\mathrm{e}^{-P}\left(-\omega_{t}^{2}+\omega_{\theta}^{2}\right)-\mathrm{e}^{P}\left(-\chi_{t}^{2}+\chi_{\theta}^{2}\right)\right] \\
& -\omega_{t t}+\omega_{\theta \theta}=-P_{t} \omega_{t}+P_{\theta} \omega_{\theta} \\
& -\chi_{t t}+\chi_{\theta \theta}=-\left[-P_{t} \chi_{t}+P_{\theta} \chi_{\theta}\right] \\
& \lambda_{t}=t\left(P_{t}^{2}+P_{\theta}^{2}\right)+4\left[\mathrm{e}^{-P}\left(\omega_{t}^{2}+\omega_{\theta}^{2}\right)+\mathrm{e}^{P}\left(\chi_{t}^{2}+\chi_{\theta}^{2}\right)\right] \\
& \lambda_{\theta}=2 t P_{t} P_{\theta}+8\left(\mathrm{e}^{-P} \omega_{t} \omega_{\theta}+\mathrm{e}^{P} \chi_{t} \chi_{\theta}\right) \\
& \omega_{t} \chi_{t}=\omega_{\theta} \chi_{\theta} .
\end{aligned}
$$

The full set of Einstein equations includes one involving the second derivatives of $\lambda$. It is a consequence of (6) and (7) and plays a minor role in what follows. It is written down in terms of other variables later. Equation (8) is the consistency condition alluded to in the previous section. The only large classes of solutions known which are consistent with this equation are those where either $\omega$ or $\chi$ vanishes. In [4], the authors considered other possibilities but this did not lead to the discovery of other interesting classes of solutions. In most of what follows it will be assumed that $\omega=0$. Assuming $\chi=0$ would lead to an equivalent problem. These two cases are related by interchanging the roles of the two Killing vectors.

Equations (3), (5), (6) and (7) with $\omega=0$ can be shown to be equivalent to the equations for (not necessarily polarized) Gowdy vacuum spacetimes by introducing new variables. These are given by

$$
\begin{aligned}
& \bar{P}=\frac{1}{2}(P-\log t) \\
& \bar{\lambda}=\frac{1}{4}(\lambda-\log t)-\bar{P} .
\end{aligned}
$$

Then the equations for $\bar{P}, \chi$ and $\lambda$ coming from (3), (5), (6) and (7) are the basic equations for Gowdy vacuum spacetimes (cf ([16]) with $P$ replaced by $\bar{P}, Q$ replaced by $\chi$ and $\lambda$ replaced by $\bar{\lambda}$. These equations are

$$
-\bar{P}_{t t}-t^{-1} \bar{P}_{t}+\bar{P}_{\theta \theta}=\mathrm{e}^{2 \bar{P}}\left(-\chi_{t}^{2}+\chi_{\theta}^{2}\right)
$$




$$
\begin{aligned}
& -\chi_{t t}-t^{-1} \chi_{t}+\chi_{\theta \theta}=-2\left(-\bar{P}_{t} \chi_{t}+\bar{P}_{\theta} \chi_{\theta}\right) \\
& \bar{\lambda}_{t}=t\left[\bar{P}_{t}^{2}+\bar{P}_{\theta}^{2}+\mathrm{e}^{2 \bar{P}}\left(\chi_{t}^{2}+\chi_{\theta}^{2}\right)\right] \\
& \bar{\lambda}_{\theta}=2 t\left[\bar{P}_{t} \bar{P}_{\theta}+\mathrm{e}^{2 \bar{P}} \chi_{t} \chi_{\theta}\right] .
\end{aligned}
$$

In terms of these variables the metric is

$$
\mathrm{e}^{2(\bar{\lambda}+\bar{P})}\left(-\mathrm{d} t^{2}+\mathrm{d} \theta^{2}\right)+t^{2} \mathrm{e}^{2 \bar{P}} \mathrm{~d} x^{2}+\mathrm{e}^{-2 \bar{P}} \mathrm{~d} y^{2} .
$$

The additional equation involving the second derivative of $\bar{\lambda}$ reads

$$
-\bar{\lambda}_{t t}-t^{-1} \bar{\lambda}_{t}+\bar{\lambda}_{\theta \theta}=2\left(\bar{P}_{\theta}^{2}+\mathrm{e}^{2 \bar{P}} \chi_{\theta}^{2}\right)
$$

\section{Structure of the singularity}

Since the equations for solutions of the Einstein-Maxwell system with polarized Gowdy symmetry are identical to the well-known Gowdy equations in the vacuum case, it can be concluded from a theorem of [10] that the solution corresponding to any initial data with constant $R$ exists globally on the interval $(0, \infty)$ when expressed in areal time. The purpose of this section is to investigate the asymptotics of these solutions in the limit $t \rightarrow 0$. Interpreting a result of [17] in terms of the present problem shows that for a fixed value of $\theta$ the quantity $t\left[\left(\bar{P}_{t}^{2}+\mathrm{e}^{2 \bar{P}} \chi_{t}^{2}\right)(t, \theta)\right]^{\frac{1}{2}}$ converges to a limit $v_{\infty}(\theta)$ as $t \rightarrow 0$. This quantity is called the asymptotic velocity at $\theta$. It turns out to be useful to examine regions $\mathcal{D}_{\theta_{0}}$ defined as the set of points $(t, \theta)$ such that $\left|\theta-\theta_{0}\right| \leqslant t$. Intuitively this can be thought of as the causal future of a point of the singularity defined by $\theta=\theta_{0}$.

The structure of the singularity will be described in detail for a generic set $\mathcal{G}_{c}$ of initial data, which is strictly analogous to a similar set introduced in [17], using the relation between vacuum solutions and polarized Einstein-Maxwell solutions. By analogy with [17] we can define what is meant by a solution having a non-degenerate false spike or a non-degenerate true spike at some value of $\theta$. The detailed definition will not be reproduced here. For convenience call a point $\theta$ an ordinary point for a given solution if $0<v_{\infty}(\theta)<1$ and $\lim _{t \rightarrow 0} t P_{t}(t, \theta)=-v_{\infty}(\theta)$. Define $\mathcal{G}_{l, m, c}$ to be the set of initial data determining solutions for which all points $\theta$ are ordinary except for $l$ non-degenerate false spikes and $m$ nondegenerate true spikes. Then $\mathcal{G}_{c}$ is the union of $\mathcal{G}_{l, m, c}$ over all non-negative integers $l$ and $m$. Data belonging to $\mathcal{G}_{c}$ are called generic. This subset is open in the set of initial data in the $C^{0} \times C^{1}$ topology and dense in the $C^{\infty}$ topology [19]. A comment should be made on the subscript $c$ which is included here for consistency with Ringström's notation. It is a reminder that initial data for the Gowdy equations must satisfy an integral constraint for topological reasons. An analogous constraint occurs in the Einstein-Maxwell case. It arises by integrating equation (14) with respect to $\theta$. The integral of the left-hand side vanishes due to periodicity and so the integral of the right-hand side must vanish too. If there is a false spike at $\theta$ then $0<v_{\infty}(\theta)<1$ while if there is a true spike $1<v_{\infty}(\theta)<2$. Thus, in particular, generic data are such that $v_{\infty}$ is never equal to 1 at any point. In the vacuum case, this statement is enough to imply blow-up of the Kretschmann scalar along any past-directed causal curve due to proposition 1.19 of [17].

To prove inextendibility it will be necessary to consider the case of normal points, false spikes and true spikes separately. First the case of a normal point will be examined. The expression for the Kretschmann scalar is unwieldy, and it might be thought that it would be easier to prove inextendibility in the electromagnetic case by showing that the invariant $F_{\alpha \beta} F^{\alpha \beta}$ blows up. Unfortunately this turns out not to be the case. There is a large class of 
Gowdy solutions (the low velocity solutions) which, when translated to the case of interest here, admit asymptotic expansions of the following form:

$$
\begin{aligned}
& \bar{P}(t, \theta)=-v_{\infty}(\theta) \log t+\cdots \\
& \chi(t, \theta)=q(\theta)+\psi(\theta) t^{2 v_{\infty}}+\cdots,
\end{aligned}
$$

where the terms omitted are lower order as $t \rightarrow 0$. It follows that $\mathrm{e}^{-2 \bar{\lambda}}$ is proportional to $t^{-2 v_{\infty}^{2}}$ in leading order while

$$
\chi_{\theta}^{2}-\chi_{t}^{2}=q_{\theta}^{2}-4 v_{\infty}^{2} t^{4 v_{\infty}-2} \psi^{2}+\cdots
$$

Since

$$
F^{\alpha \beta} F_{\alpha \beta}=2 \mathrm{e}^{-2 \bar{\lambda}}\left(\chi_{\theta}^{2}-\chi_{t}^{2}\right),
$$

it follows that the information contained in the above expansions and the fact that $0<v_{\infty}<1$ do not always suffice to determine whether the invariant blows up or not. For if $v_{\infty}=\frac{1}{2}$ and $q_{\theta}^{2}=4 v_{\infty}^{2} \psi^{2}$, the leading term in the expansion for $F^{\alpha \beta} F_{\alpha \beta}$ vanishes and whether the next to leading term blows up cannot be decided using the available information. For the solutions considered here $F^{\alpha \beta *} F_{\alpha \beta}=0$ and so this invariant is not helpful.

Next the Kretschmann scalar will be examined. An observation which simplifies the calculations is that, due to the discrete symmetries of the spacetimes under consideration, a component $R_{\alpha \beta \gamma \delta}$ which does not vanish must have an even number of indices equal to 2 and an even number equal to 3 . It follows that up to symmetries (not including the algebraic Bianchi identity) the only components which may be nonzero are

$$
R_{0101}, R_{0202}, R_{0212}, R_{1212}, R_{0303}, R_{0313}, R_{1313}, R_{2323} \text {. }
$$

The Kretschmann scalar is equal to

$$
\begin{aligned}
4\left[R_{0101} R^{0101}+\right. & R_{0202} R^{0202}+R_{1212} R^{1212}+R_{0303} R^{0303} \\
& \left.+R_{1313} R^{1313}+R_{2323} R^{2323}\right]+8\left[R_{0212} R^{0212}+R_{0313} R^{0313}\right] .
\end{aligned}
$$

In terms of the metric coefficients the individual terms are given by

$$
\begin{aligned}
& R_{0101} R^{0101}=t^{-4} \mathrm{e}^{-4(\bar{\lambda}+\bar{P})}\left[-t^{2} \bar{\lambda}_{t t}-t^{2} \bar{P}_{t t}+t^{2}\left(\bar{P}_{\theta \theta}+\bar{\lambda}_{\theta \theta}\right)\right]^{2} \\
& R_{0202} R^{0202}=t^{-4} \mathrm{e}^{-4(\bar{\lambda}+\bar{P})}\left[t \bar{\lambda}_{t}+t^{2} \bar{P}_{t} \bar{\lambda}_{t}-t^{2} \bar{P}_{t t}-t \bar{P}_{t}+t^{2}\left(\bar{P}_{\theta}^{2}+\bar{P}_{\theta} \bar{\lambda}_{\theta}\right)\right]^{2} \\
& R_{1212} R^{1212}=t^{-4} \mathrm{e}^{-4(\bar{\lambda}+\bar{P})}\left[t \bar{\lambda}_{t}+t^{2} \bar{\lambda}_{t} \bar{P}_{t}+t \bar{P}_{t}+t^{2} \bar{P}_{t}^{2}-t^{2}\left(\bar{P}_{\theta \theta}-\bar{P}_{\theta} \bar{\lambda}_{\theta}\right)\right]^{2} \\
& R_{0303} R^{0303}=t^{-4} \mathrm{e}^{-4(\bar{\lambda}+\bar{P})}\left[t^{2} \bar{P}_{t t}-t^{2} \bar{P}_{t} \bar{\lambda}_{t}-2 t^{2} \bar{P}_{t}^{2}-t^{2}\left(\bar{P}_{\theta} \bar{\lambda}_{\theta}+\bar{P}_{\theta}^{2}\right)\right]^{2} \\
& R_{1313} R^{1313}=t^{-4} \mathrm{e}^{-4(\bar{\lambda}+\bar{P})}\left[-t^{2} \bar{P}_{t} \bar{\lambda}_{t}-t^{2} \bar{P}_{t}^{2}+t^{2}\left(\bar{P}_{\theta \theta}-2 \bar{P}_{\theta}^{2}-\bar{P}_{\theta} \bar{\lambda}_{\theta}\right)\right]^{2} \\
& R_{2323} R^{2323}=t^{-4} \mathrm{e}^{-4(\bar{\lambda}+\bar{P})}\left[-t \bar{P}_{t}-t^{2} \bar{P}_{t}^{2}+t^{2} \bar{P}_{\theta}^{2}\right]^{2} \\
& R_{0212} R^{0212}=-t^{-4} \mathrm{e}^{-4(\bar{\lambda}+\bar{P})}\left[-t \bar{\lambda}_{\theta}-t^{2} \bar{P}_{t} \bar{P}_{\theta}+t^{2} \bar{P}_{t \theta}-t^{2} \bar{P}_{\theta} \bar{\lambda}_{t}-t^{2} \bar{\lambda}_{\theta} \bar{P}_{t}\right]^{2} \\
& R_{0313} R^{0313}=-t^{-4} \mathrm{e}^{-4(\bar{\lambda}+\bar{P})}\left[-t^{2} \bar{P}_{t \theta}+t^{2} \bar{P}_{t} \bar{\lambda}_{\theta}+t^{2} \bar{P}_{\theta} \bar{\lambda}_{t}+3 t^{2} \bar{P}_{t} \bar{P}_{\theta}\right]^{2} .
\end{aligned}
$$

The first six terms are manifestly non-negative while the last two are manifestly non-positive. The fact that all terms contain a common factor helps when comparing their magnitudes. It suffices to compare the magnitudes of the quantities in square brackets. Suppose it can be shown that in a given solution at least one of the positive terms tends to infinity as $t \rightarrow 0$ and that both the negative terms are negligible with respect to that positive term. Then this implies that the Kretschmann scalar tends to infinity. It turns out, as will be explained in more detail later, that for this question the spatial derivatives in these expressions are unimportant 
compared to the time derivatives. The essential information needed for the proof is contained in the following expressions:

$$
\begin{aligned}
& R_{0101} R^{0101}=t^{-4} \mathrm{e}^{-4(\bar{\lambda}+\bar{P})}\left[-t^{2} \bar{\lambda}_{t t}-t^{2} \bar{P}_{t t}\right]^{2}+\cdots \\
& R_{0202} R^{0202}=t^{-4} \mathrm{e}^{-4(\bar{\lambda}+\bar{P})}\left[t \bar{\lambda}_{t}+t^{2} \bar{P}_{t} \bar{\lambda}_{t}-t^{2} \bar{P}_{t t}-t \bar{P}_{t}\right]^{2}+\cdots \\
& R_{1212} R^{1212}=t^{-4} \mathrm{e}^{-4(\bar{\lambda}+\bar{P})}\left[t \bar{\lambda}_{t}+t^{2} \bar{\lambda}_{t} \bar{P}_{t}+t \bar{P}_{t}+t^{2} \bar{P}_{t}^{2}\right]^{2}+\cdots \\
& R_{0303} R^{0303}=t^{-4} \mathrm{e}^{-4(\bar{\lambda}+\bar{P})}\left[t^{2} \bar{P}_{t t}-t^{2} \bar{P}_{t} \bar{\lambda}_{t}-2 t^{2} \bar{P}_{t}^{2}\right]^{2}+\cdots \\
& R_{1313} R^{1313}=t^{-4} \mathrm{e}^{-4(\bar{\lambda}+\bar{P})}\left[-t^{2} \bar{P}_{t} \bar{\lambda}_{t}-t^{2} \bar{P}_{t}^{2}\right]^{2}+\cdots \\
& R_{2323} R^{2323}=t^{-4} \mathrm{e}^{-4(\bar{\lambda}+\bar{P})}\left[-t \bar{P}_{t}-t^{2} \bar{P}_{t}^{2}\right]^{2}+\cdots \\
& R_{0212} R^{0212}=\cdots \\
& R_{0313} R^{0313}=\cdots
\end{aligned}
$$

The terms not explicitly written in these equations are not relevant for the proof which follows. It is not claimed that in the approach to the singularity each of the terms which have been omitted is small in comparison to the terms which have been written out explicitly.

Consider now the case of a normal point $\theta_{0}$. Then proposition 1.5 of [17] provides information about the limiting behaviour of the solution in a neighbourhood of $\theta_{0}$. It implies that $t \bar{P}_{t}$ converges to $-v_{\infty}$ and that $t \bar{P}_{\theta}$ and $t^{2} \bar{P}_{t \theta}$ tend to zero. It also shows that if equations (13) and (14) are multiplied by $t$, the terms containing derivatives of $\chi$ on the right-hand sides tend to zero as $t \rightarrow 0$. If follows that $t \bar{\lambda}_{t}$ converges to $v_{\infty}^{2}$ and $t \bar{\lambda}_{\theta}$ tends to zero as $t \rightarrow 0$. These facts together show that the quantities in square brackets in the expressions for $R_{0212} R^{0212}$ and $R_{0313} R^{0313}$ tend to zero at the singularity. The expression in the square brackets in $R_{2323} R^{2323}$ converges to $v_{\infty}-v_{\infty}^{2} \neq 0$. The expression $\mathrm{e}^{-4(\bar{P}+\bar{\lambda})}$ is asymptotic to an expression proportional to $t^{4\left(v_{\infty}-v_{\infty}^{2}\right)}$. Since $v_{\infty}\left(1-v_{\infty}\right)<1$ this is enough to conclude that the curvature blows up uniformly in a neighbourhood of a normal point $\theta_{0}$. It is also part of the conclusions of proposition 1.5 of [17] that if $\theta_{0}$ is a false spike, a change of variables can be carried out which gives a new solution with the asymptotic properties which hold for a normal point. Since it is known that this transformation does not change the spacetime geometry but only the way it is represented the curvature properties are unaffected. Thus curvature blow-up also holds in the case of a false spike. It remains to treat the case of a true spike at a point $\theta_{0}$. In that case, proposition 6.7 of [17] implies that $t \bar{P}_{\theta}$ and $t \mathrm{e}^{\bar{P}} \chi_{\theta}$ converge to zero uniformly on $\mathcal{D}_{\theta_{0}}$. Corollary 6.8 shows that $t^{2} P_{t \theta}$ also converges to zero there. By proposition 6.11 of [17] and the remark following it $t \bar{P}_{t}$ and $t \mathrm{e}^{\bar{P}} \chi_{t}$ converge to $-v_{\infty}$ and zero respectively on that region. In this case $v_{\infty}\left(1-v_{\infty}\right)<0$. Thus the Kretschmann scalar can be controlled as in the other cases, with the difference that the control is only obtained on the region $\mathcal{D}_{\theta_{0}}$ rather than for an interval of $\theta$ containing $\theta_{0}$.

In the following theorem, the topology on the set of initial data used is the $C^{\infty}$ topology.

Theorem 1. There is an open dense subset $\mathcal{G}_{c}$ of the set of smooth initial data for the EinsteinMaxwell equations with polarized Gowdy symmetry and constant areal time such that the Kretschmann scalar tends to infinity along any inextendible past-directed causal geodesic.

Proof. The set $\mathcal{G}_{c}$ has been defined above. For a solution evolving from data belonging to this set each point $\theta_{0}$ is either an ordinary point, a false spike or a true spike. It has been shown that in each of these three cases the Kretschmann scalar blows up uniformly on $\mathcal{D}_{\theta_{0}}$ as $t \rightarrow 0$. It is shown in [17] that any inextendible past-directed causal geodesic eventually lies in a region of this type with some value of $\theta_{0}$ as $t \rightarrow 0$. The proof of this is pure Lorentzian geometry and makes no use of the Einstein equations. Hence it also applies to the situation of this theorem. 


\section{Future geodesic completeness}

This section is concerned with the asymptotic behaviour of solutions in future, i.e. with the behaviour as the areal time $t$ tends to infinity. The asymptotic behaviour of solutions of the vacuum Gowdy equations has been determined in detail in [16]. This information suffices in particular to prove future geodesic completeness in the vacuum case. It will be shown that similar arguments can be used to give a proof of future geodesic completeness of solutions of the Einstein-Maxwell equations with polarized Gowdy symmetry.

As in the vacuum case, it is convenient in the proof of geodesic completeness to distinguish between homogeneous and inhomogeneous spacetimes. The homogeneous case is that in which $\bar{P}, \chi$ and $\bar{\lambda}$ depend only on $t$. Geodesic completeness in the homogeneous case follows from theorem 2.1 of [13]. That theorem applies to solutions of the Einstein equations coupled to a general class of matter models which satisfy two conditions: the dominant energy condition and the non-negative sum pressures condition. Both of these hold for the Maxwell field. In fact the second follows from the first because the energy-momentum tensor of the Maxwell field has vanishing trace. It should also be noted that for these solutions, which are of Bianchi type I, the mean curvature of the hypersurfaces of constant $t$ is everywhere negative and hence, in particular, bounded above.

It remains to consider the inhomogeneous case. From proposition 1.8 of [16], it can be concluded that $\bar{P}_{t}, \bar{P}_{\theta}$, $\mathrm{e}^{\bar{P}} \chi_{t}$ and $\mathrm{e}^{\bar{P}} \chi_{\theta}$ are $O\left(t^{-1 / 2}\right)$ as $t \rightarrow \infty$, uniformly in $\theta$. This means in particular that $\bar{\lambda}_{t}$ is bounded in future. From theorem 1.7 of [16], it follows that $\bar{\lambda}=c_{\bar{\lambda}} t+O(\log t)$ for a positive constant $c_{\bar{\lambda}}$ as $t \rightarrow \infty$. It is at this point that the condition that the solution is inhomogeneous is used. Combining these facts shows that $\bar{\lambda}+\bar{P}=c_{\bar{\lambda}} t+O\left(t^{\frac{1}{2}}\right)$. Define an orthonormal frame $\left\{e_{\mu}\right\}$ by normalizing the coordinate basis vectors $\frac{\partial}{\partial t}, \frac{\partial}{\partial \theta}, \frac{\partial}{\partial x}, \frac{\partial}{\partial y}$. A computation shows that the corresponding rotation coefficients satisfy an inequality of the form

$$
\left|g\left(\nabla_{e_{\mu}} e_{\nu}, e_{\rho}\right)\right| \leqslant C t^{-\frac{1}{2}} \mathrm{e}^{-\bar{\lambda}-\bar{P}}
$$

for a constant $C$ whenever at least two of the indices $(\mu, v, \rho)$ are equal to 2 or 3 . The facts just listed suffice to show that the argument used to prove geodesic completeness in the vacuum case in [16] also applies to the Einstein-Maxwell case, proving geodesic completeness in that case too.

In [16] further information about the late-time behaviour of Gowdy spacetimes is derived. It is shown that, remarkably, there are solutions with oscillations of $P$ which persist for all times. More precisely, the amplitude of the oscillation does not decay and the fact that $P_{t}$ converges to zero is explained by the fact that the frequency of the oscillation decreases with time. The derivative $P_{\theta}$ does decay, which means that in a sense the solution becomes homogeneous asymptotically. Nevertheless the dynamics of these solutions is different from that of any homogeneous solution. These solutions can be used to produce solutions of the Einstein-Maxwell equations by the correspondence which is central to this paper. The statements made about $P$ in the vacuum case apply directly to $\bar{P}$ in the electromagnetic case. Considering $\chi$ it is seen that the electromagnetic potential oscillates with an amplitude which does not decay while the decrease in the frequency of the oscillation leads to decay of the electric field. The potential becomes spatially homogeneous asymptotically and this gives decay of the magnetic field.

A key step in proving the results about future asymptotics in the vacuum case is to show that the following energy-like quantity tends to zero as $t \rightarrow \infty$ :

$$
\mathcal{E}_{1}(t)=\frac{1}{2} \int_{S^{1}}\left[P_{t}^{2}+P_{\theta}^{2}+\mathrm{e}^{2 P}\left(Q_{t}^{2}+Q_{\theta}^{2}\right)\right](t, \theta) \mathrm{d} \theta .
$$


Of course there is a corresponding quantity in the Einstein-Maxwell case which also tends to zero as $t \rightarrow \infty$. It is given explicitly by

$$
\mathcal{E}_{2}(t)=\frac{1}{2} \int_{S^{1}}\left[\bar{P}_{t}^{2}+\bar{P}_{\theta}^{2}+\mathrm{e}^{2 \bar{P}}\left(\chi_{t}^{2}+\chi_{\theta}^{2}\right)\right](t, \theta) \mathrm{d} \theta .
$$

\section{Conclusions and outlook}

Given a matter model in general relativity for which the Einstein-matter equations have a well-posed initial value problem there exists a maximal Cauchy development corresponding to each initial data set. The strong cosmic censorship hypothesis says that for generic initial data this maximal development is not extendible to a larger spacetime. There are some technical choices involved in this definition: how much regularity is assumed for the data, what topology is put on these data and what regularity properties are assumed of an extension. Here no general discussion of these issues will be given-we will just make one reasonable choice. It is assumed that the data are smooth, i.e. $C^{\infty}$. They are topologized using the $C^{\infty}$ topology, i.e. the topology of uniform convergence of a geometrical object together with its derivatives of all orders. Extendibility is taken in the sense of extensions of the geometry of class $C^{2}$. It should be noted that since cosmic censorship is such a hard problem, it is common to consider the related problem where the initial data is generic subject to some extra restrictions, e.g. symmetry restrictions. This is the case in the results of this paper.

The main theorem of the paper can now be given. It is a statement about strong cosmic censorship for the class of spacetimes involved.

Theorem 2. For data belonging to the open dense subset $\mathcal{G}_{c}$ of the set of smooth initial datafor the Einstein-Maxwell equations with polarized Gowdy symmetry and constant area radius, the corresponding maximal Cauchy development is inextendible.

Proof. Let $(g, A)$ be initial data belonging to $\mathcal{G}_{c}$. By the results of section 4 the maximal Cauchy development of these data is inextendible towards the past since the Kretschmann scalar is unbounded along any inextendible past-directed causal geodesic. By the results of section 5, the maximal development is future geodesically complete and hence inextendible towards the future. This completes the proof.

In fact inextendibility towards the future already follows from global existence to the future in areal time, as has been shown in [6]. It is nevertheless good that more has been proved about the future asymptotics since this gives more insight into what is going on. It should also be noted that the argument using global existence in areal time is fundamentally restricted to spacetimes with symmetry while proving geodesic completeness is a strategy which can in principle be applied to situations without any symmetry.

Note that the fact that the above theorem deals only with initial data with constant area radius is not a serious restriction since any solution admits a Cauchy hypersurface carrying data of this type.

In this paper, the dynamics of solutions of the Einstein-Maxwell equations with polarized Gowdy symmetry has been analysed rather comprehensively. This was based on the existing analysis of the dynamics of vacuum Gowdy spacetimes which need not be polarized. This raises the obvious question, whether it is possible to do something similar for general solutions of the Einstein-Maxwell equations with Gowdy symmetry. Some aspects of this problem will now be discussed. In the full Einstein-Maxwell case, there are two metric coefficients $P$ and $Q$ as in the vacuum case and two components $\omega$ and $\chi$ of the potential. 
In looking at the behaviour of the solutions in the past, a possible starting point is to try to extend the results on the existence of low velocity solutions of the Gowdy equations proved in [9] using Fuchsian methods. A more complicated system of this type, the EinsteinMaxwell-dilaton-axion system was discussed from this point of view in [11]. Setting the dilaton, the axion and the dilaton coupling constant in that system to zero gives rise to the Einstein-Maxwell system. A special case considered in [11] is in principle relevant to this but unfortunately it is not self-consistent in the non-polarized case since one of the field equations was neglected (cf the remarks on $\mathrm{p} 166$ of [15]). Other special cases considered in [11] may provide an avenue to a greater understanding of singularities of solutions of the full Einstein-Maxwell-dilaton-axion system. In any case, the question of whether Fuchsian techniques can be used to obtain solutions of the Einstein-Maxwell equations with Gowdy symmetry depending on the full number of free functions (i.e. eight) remains open.

Global existence in the future in areal time is known and this suffices to prove the part of strong cosmic censorship which concerns the future evolution using the arguments of [6]. On the other hand, the detailed asymptotics have not been determined. Parts of the argument which applies to the vacuum Gowdy case have been extended to the Einstein-Maxwell case in [18]. These are the parts which concern the decay of an energy-like quantity analogous to those defined in (24) and (25). However other important parts of what was done in the vacuum case have not yet been generalized.

These remarks show that the task of extending the results on the Einstein-Maxwell equations with polarized Gowdy symmetry obtained in this paper to the case of solutions with fully general Gowdy symmetry presents some interesting challenges.

\section{Appendix A. The dominant energy condition for the Maxwell field}

It is common knowledge that the Maxwell field satisfies the dominant energy condition but it is difficult to find a concise, elementary and self-contained proof in the literature. For the convenience of the reader one will be included here. The dominant energy condition for an energy-momentum tensor $T^{\alpha \beta}$ can be formulated as the condition that $T_{\alpha \beta} v^{\alpha} w^{\beta} \geqslant 0$ for all future-pointing causal vectors $v^{\alpha}$ and $w^{\beta}$. Since any future-pointing causal vector can be written as a sum of two future-pointing null vectors, it suffices to verify the condition in the case that $v^{\alpha}$ and $w^{\alpha}$ are equal to future-pointing null vectors $l^{\alpha}$ and $n^{\alpha}$. By possibly rescaling $l^{\alpha}$ and adding two spacelike vectors $x^{\alpha}$ and $y^{\alpha}$ these may be completed to a null basis where the vectors have the following inner products: $l_{\alpha} n^{\alpha}=-1, l^{\alpha} x_{\alpha}=l^{\alpha} y_{\alpha}=n^{\alpha} x_{\alpha}=n^{\alpha} y_{\alpha}=0$, $x^{\alpha} x_{\alpha}=y^{\alpha} y_{\alpha}=1, x^{\alpha} y_{\alpha}=0$. The electromagnetic field can be expanded in this basis to give

$$
T_{\alpha \beta}=A l_{[\alpha} n_{\beta]}+B l_{[\alpha} x_{\beta]}+C l_{[\alpha} y_{\beta]}+D x_{[\alpha} y_{\beta]}+E n_{[\alpha} x_{\beta]}+G n_{[\alpha} y_{\beta]},
$$

where the coefficients are constants. Straightforward computations give

$F_{\alpha \gamma} F_{\beta}^{\gamma} l^{\alpha} n^{\beta}=\frac{1}{4}\left(A^{2}+E B+G C\right), F_{\alpha \beta} F^{\alpha \beta}=\frac{1}{2}\left(D^{2}-A^{2}\right)-E B-G C$

and

$$
T_{\alpha \beta} l^{\alpha} n^{\beta}=\frac{1}{32 \pi}\left(A^{2}+D^{2}\right)
$$

\section{References}

[1] Andréasson H, Rein G and Rendall A D 2003 On the Einstein-Vlasov system with hyperbolic symmetry Math. Proc. Camb. Phil. Soc. 134 529-49

[2] Andréasson H, Rendall A D and Weaver M 2004 Existence of CMC and constant areal time foliations in $T^{2}$ symmetric spacetimes with Vlasov matter Commun. Partial Diff. Eqns 29 237-62 
[3] Berger B, Chruściel P T, Isenberg J and Moncrief V 1997 Global foliations of vacuum spacetimes with $T^{2}$ isometry Ann. Phys., NY $260117-48$

[4] Carmeli M, Charach Ch and Malin S 1981 Survey of cosmological models with gravitational, scalar and electromagnetic waves Phys. Rep. 76 79-156

[5] Chruściel P T, Isenberg J and Moncrief V 1990 Strong cosmic censorship in polarised Gowdy spacetimes Class. Quantum Grav. 7 1671-80

[6] Dafermos M and Rendall A D 2006 Inextendibility of expanding cosmological models with symmetry Class. Quantum Grav. 22 L143-7

[7] Dafermos M and Rendall A D 2006 Strong cosmic censorship for $T^{2}$ symmetric cosmological spacetimes with collisionless matter arXiv:gr-qc/0610075

[8] Dafermos M and Rendall A D 2007 Strong cosmic censorship for surface-symmetric cosmological spacetimes with collisionless matter arXiv:gr-qc/0701034

[9] Kichenassamy S and Rendall A D 1998 Analytic description of singularities in Gowdy spacetimes Class. Quantum Grav. 15 1339-55

[10] Moncrief V 1981 Global properties of Gowdy spacetimes with $T^{3} \times \mathbf{R}$ topology Ann. Phys., NY 132 87-107

[11] Narita M, Torii T and Maeda K 2000 Asymptotic singular behaviour of Gowdy spacetimes in string theory Class. Quantum Grav. 17 4597-613

[12] Nungesser E 2008 Strong cosmic censorship in polarized $T^{3}$-Gowdy symmetric spacetimes with a Maxwell field Diploma Thesis Free University, Berlin

[13] Rendall A D 1995 Global properties of locally spatially homogeneous cosmological models with matter Math. Proc. Camb. Phil. Soc. 118 511-26

[14] Rendall A D 1997 Existence of constant mean curvature foliations in spacetimes with two-dimensional local symmetry Commun. Math. Phys. 189 145-64

[15] Rendall A D 2008 Partial Differential Equations in General Relativity (Oxford: Oxford University Press)

[16] Ringström H 2004 On a wave map arising in general relativity Commun. Pure Appl. Math. 57 657-703

[17] Ringström H 2006 Existence of an asymptotic velocity and implications for the asymptotic behaviour in the direction of the singularity in $T^{3}$-Gowdy Commun. Pure Appl. Math. 59 977-1041

[18] Ringström H 2006 On the $T^{3}$-Gowdy symmetric Einstein-Maxwell equations Ann. Henri Poincaré 7 1-20

[19] Ringström H Strong cosmic censorship in $T^{3}$-Gowdy spacetimes Ann. Math. (at press)

[20] Smulevici J 2008 Strong cosmic censorship for $T^{2}$-symmetric spacetimes with positive cosmological constant and matter Ann. Henri Poincaré 9 1425-53

[21] Tegankong D 2005 Global existence and future asymptotic behaviour for solutions of the Einstein-Vlasov-scalar field system with surface symmetry Class. Quantum Grav. 22 2381-92

[22] Weaver M 1999 Asymptotic behaviour of solutions to Einstein's equation PhD Thesis University of Oregon 